\title{
Check-list of the vascular flora of the "Bosco di Gibilmanna", a Special Area of Conservation (S.A.C.) in northern Sicily (Italy)
}

\author{
Salvatore Cambria' \& Gianmarco Tavilla' \\ ${ }^{1}$ Department of Biological, Geological and Environmental Sciences, University of Catania, Italy \\ "Corresponding author, e-mail: cambria_salvatore@yahoo.it
}

ABSTRACT

This paper reports the results of a floristic survey carried out in the area falling within the S.A.C. ITA020002 "Boschi di Gibilmanna e Cefalù" (Sicily, Italy), a site with a marked environmental heterogeneity lying from almost sea level to over 1000 meters of altitude. On the basis of our investigations, a total of 605 taxa has been detected, including some endemic or rare species, as Anthemis cupaniana Tod. ex Nyman, Arabis collina Ten. subsp. rosea (DC.) Minuto, Brassica incana Ten., Hieracium racemosum Waldst. \& Kit. ex Willd. subsp. crinitum (Sm.) Rouy, Melittis melissophyllum L. subsp. albida (Guss.) P.W. Ball., Stachys sylvatica L., Symphytum gussonei F.W. Schultz, Viburnum tinus L., etc. A map with floristic emergencies has been prepared in order to highlight the sites with the greatest conservation interest in the area. Besides, the taxonomical, biological and chorological composition of this flora is analyzed and discussed.

KEY WORDS $\quad$ Mediterranean; Plant conservation; Endemism; Madonie.

Received 6.01.2020; accepted 11.03.2020; published online 28.04.2020.

\section{INTRODUCTION}

The Madonie massif, located in north-western Sicily, represents one of the main hotspots of plant biodiversity in the Mediterranean area, hosting about 1500 taxa and 170 endemisms (Raimondo et al., 2004a). The central highest part of the massif is quite well studied from a floristic and vegetational point of view (see Raimondo, 1980, 1984a, 1984b; Brullo, 1984), while specific contributions on the lower northern slopes are very scarce. Although this area does not present a phytogeographical interest comparable to that of the mountain belt, it is characterized by the presence of valuable plant communities and rare species, within a strongly anthropized and degraded context. In particular, the Gibilmanna wood, located in the hills above Cefalù, retains some inter- esting forest communities, as well as sporadic aspects of chasmophilous vegetation on the limestone outcrops and temporary ponds. The first investigations about the flora of this area date back to first half of the nineteenth century, due to Enrico Pirajno di Mandralisca (1809-1864), a nobleman with wide cultural and naturalistic interests (Domina \& Mazzola, 2005), who owned a large estate in Gibilmanna. Between the end of the nineteenth and the beginning of the twentieth century other botanists provided new data, including Michele Lojacono Pojero (1853-1919), author of a monumental Flora Sicula, and Domenico Lanza (1868-1940), who usually spent the summer time in his house of Contrada Pianetti inside the Gibilmanna wood. Later, only sporadic floristic reports about this area have been quoted by Raimondo \& Mazzola (1983), Raimondo et al., (1994, 2004b) 
and Falci \& Giardina (2004). Therefore, the compilation of a floristic list for the S.A.C. ITA020002 "Boschi di Gibilmanna e Cefalù" represents the first attempt to provide a more complete and punctual account of the plant heritage occurring in the lower belt of Madonie massif.

\section{MATERIAL AND METHODS}

The boundaries of the investigated area coincide with those of the S.A.C. ITA020002 "Boschi di Gibilmanna e Cefalù" (Sicily, Italy), covering a surface of $2570 \mathrm{ha}$. It includes a quite heterogenous territory from the low hills above Cefalù at $150-200 \mathrm{~m}$ of altitude until the top of Pizzo Sant'Angelo at 1081 $\mathrm{m}$. From the geological point of view, it is mainly constituted by substrata belonging to Numidian Flysch formation, mixed with scattered limestone outcrops. The flyschoid areas are characterized by gentle and rounded morphologies, while the calcareous ones show sharp ridges, deep valleys and macro- or micro-karst microforms. Until the second post-war period this area was probably intensely cultivated at least up to $600-700 \mathrm{~m}$ above sea level, while subsequently it became a popular holiday resort both for its proximity to Cefalù and for the particularly favorable climatic conditions in summer (Palmeri, 2007). Actually, the lower stands shows a spreading urbanization, sometimes interrupted by small patches of scrub with Spartium junceum and Bupleurum fruticosum belonging to Spartio juncei-Bupleuretum fruticosi Raimondo \& Ilardi 2009 and few relicts of Pinus pinea forests (Cisto cretici-Pinetum pineae Brullo, Minissale, Siracusa, Scelsi, Spampinato 1993), often replaced by low garrigues with Cistus creticus and Cistus salvifolius. The quite evolved soils with loose texture are instead colonized by communities dominated by Quercus suber, which represent impoverished and thermophilous aspects of the Genisto aristato-Quercetum suberis Brullo 1983. The cork oak woods are widespread from 150 to 650 $\mathrm{m}$, although occupy fairly small areas and often appear in a very degraded condition. Above $500 \mathrm{~m}$, they are gradually replaced by deciduous oak woods dominated by Quercus virgiliana, often characterized by a dense undergrowth with Erica arborea and $\mathrm{Ar}$ butus unedo. This community, referable to Erico arboreae-Quercetum virgilianae Brullo \& Marcenò 1985 , still covers large surfaces in correspondence with flat or slightly inclined surfaces with deep and humid soils (Figs. 1, 2). It lacks only in the slopes with a marked inclination and shallow soils, where it is replaced by a dense woody community with Quercus ilex, generally spread from $600 \mathrm{~m}$ to the top of Pizzo Sant'Angelo (Fig. 3). Sometimes, the woody vegetation is replaced by less evolved stages belonging to the same dynamic series, as perennial grasslands and shrublands. Among the other relevant plant communities, the greater example of chasmophilous vegetation occurs in the calcareous rocks of Rocca San Nicola (Fig. 4), while an interesting temporary pond is located near the road between Gibilmanna and Gratteri. Finally, some places (e.g., contrada Romito and Colombo) are characterized by artificial woods with Castanea sativa, now abandoned and severely damaged by fungal diseases, and small reforestations with Pinus sp. pl. or less frequently also Eucalyptus sp. pl.

The field surveys for this floristic research have been carried out during the years 2012-2019 in different seasons. The nomenclature follows Bartolucci et al. (2018) and Galasso et al. (2018). Besides, Flora of Italy (Pignatti, 1982; 2017-2019), Med-Checklist (Greuter et al., 1984; 1986) and Flora Europaea (Tutin et al., 1964-1980) were also consulted. The collected samples are preserved in the herbarium of the Department of Biological, Geological and Environmental Sciences of Catania University (CAT).

This study regards the native and naturalized vascular flora, highlighting the species of particular phytogeographic interest, whose location has been indicated with GIS mapping.

\section{RESULTS AND DISCUSSION}

Our investigations have allowed to record 605 species and intraspecific taxa of vascular plants, native or naturalized. The pteridophytic component is represented by 11 taxa, while only one conifer has been detected. The angiosperms are clearly more numerous, being represented by 593 taxa. Among the dicotyledons, the richest families are Asteraceae (79 taxa), Fabaceae (61), Caryophyllaceae (28), Lamiaceae (26), Apiaceae (24) and Brassicaceae (17). The monocotyledons are represented mainly by Poaceae (44), Orchidaceae (35) and Liliaceae (17). The biological spectrum (Fig. 5) reveals the 


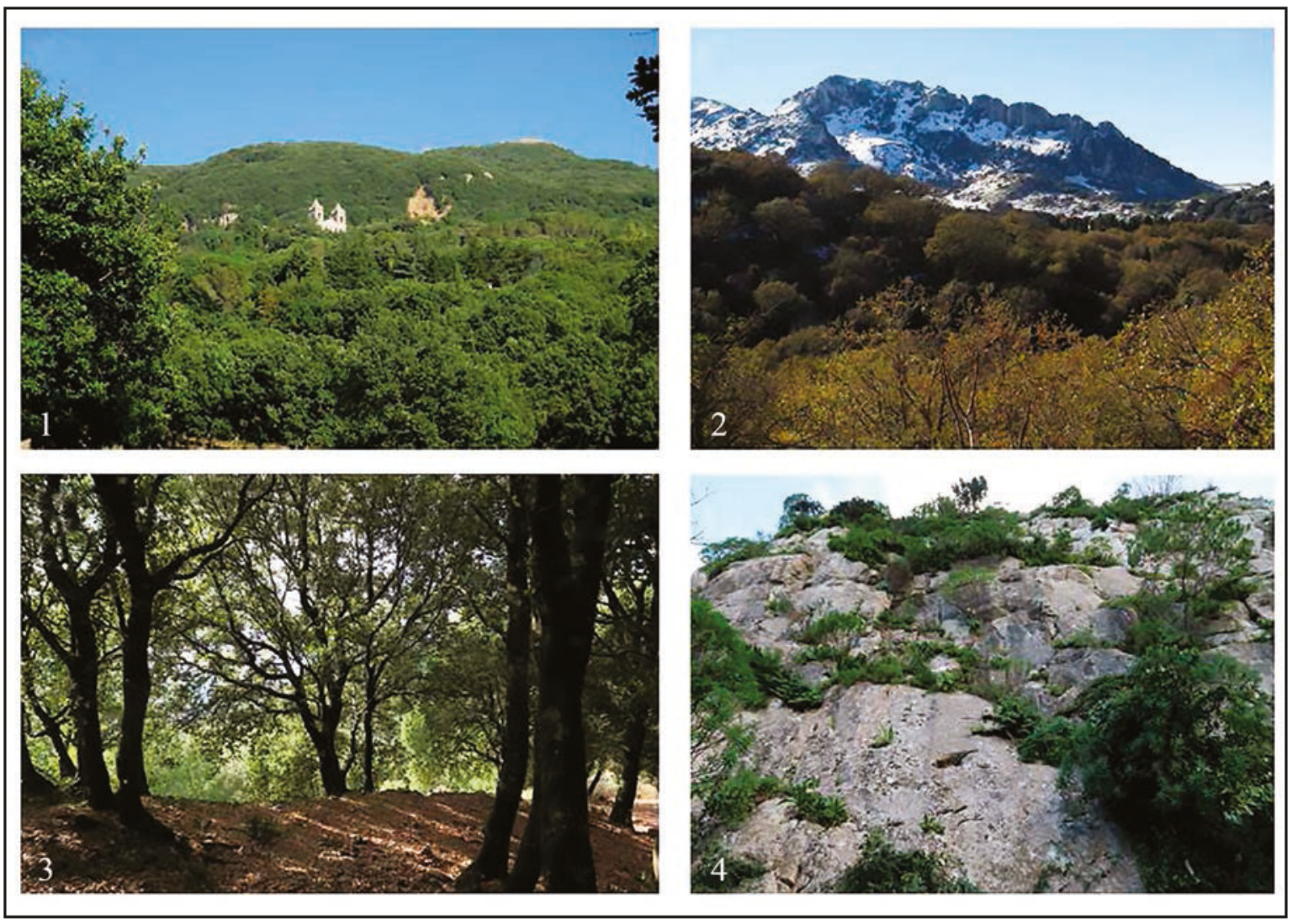

Figures 1-4. S.A.C. ITA020002 "Boschi di Gibilmanna e Cefalù" (Sicily, Italy). Figs. 1, 2: View of the woods around the Gibilmanna sanctuary. Fig. 3: Holm oak wood near Pizzo Sant'Angelo. Fig. 4: Limestone cliffs near Rocca San Nicola.

prevalence of terophytes (38\%), followed by hemicryptophytes (29\%), geophytes (18\%), phanerophytes $(7 \%)$, chamaephytes $(4 \%)$, nanophanerophytes $(3 \%)$ and hydrophytes $(1 \%)$.

As regards the chorotypes (Fig. 6), the Stenomediterranean is the most frequent $(30 \%)$, while the Eurimediterranean represents the $19 \%$. Among others, the Cosmopolitan (6\%), Eurasian (5\%), Paleotemperate (5\%), South Mediterranean (3\%), Mediterranean-Turanian (3\%), West Mediterranean $(3 \%)$, Sub-cosmopolitan $(2 \%)$, Eastern Mediterranean (2\%), European-Caucasian (2\%) and Circumboreal (2\%) can be mentioned. A special mention must be given to the alien species, which represent only the $4 \%$ of the total flora, including 12 terophytes, 6 geophytes, 4 phanerophytes and 1 hemicryptophyte. The endemic elements are represented by 32 taxa $(5 \%)$, including all species restricted to Sicily, Italy or a few neighboring areas. In particular, the Sicilian endemisms are Anthemis cupaniana, Bellevalia dubia subsp. dubia, Dianthus siculus, Echium italicum subsp. siculum, Odontites vernus subsp. siculus, $O$. archimedea, Ophrys lunulata, Pimpinella gussonei, Symphytum gussonei (Fig. 9) and Trifolium bivonae.

In addition, to the endemic species, the flora of the investigated area is characterized by some rare taxa or with a phytogeographical interest, such as Astragalus echinatus, Isoëtes durieui, Lavandula stoechas subsp. stoechas, Melittis melissophyllum L. albida, Serapias nurrica subsp. nurrica, Silene fruticosa, Viburnum tinus subsp. tinus (Fig. 7), whose local distribution is shown in figure 11. As regards the latter, during the present study a small population almost certainly indigenous was found in Contrada Romito along a small stream inside the holm oak wood. A particularly interesting group from a phytogeographic point of view is represented by the species that in this area reach the western limit of their range in Sicily, such as Brassica incana, Bupleurum fruticosum, Hieracium racemosum subsp. crinitum (Fig. 10) and Stachys sylvatica 


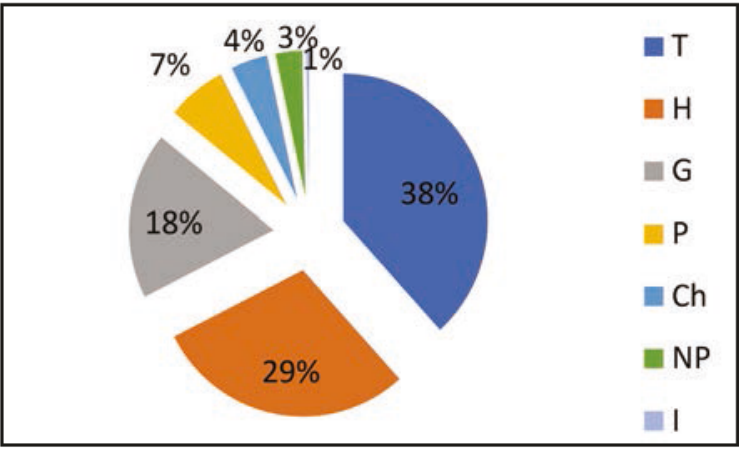

Figure 5. Biological spectrum of the vascular flora.

(Fig. 8). In fact, the Gibilmanna wood shows some intermediate features between the Madonita and Nebrodense districts from an ecological, vegetational and floristic point of view. Its woody vegetation is clearly related to that of the nearby Nebrodi mountains, also due to the common presence of flyschoid substrates and the similar climatic conditions, as well as the isolated limestone outcrops host a chasmophilous flora closer to that of the Madonie massif and in general of north-western Sicily.

\section{FLORISTIC LIST}

\section{PTERIDOPHYTA}

EQUISETACEAE

Equisetum telmateia Ehrh.

\section{SELAGINELLACEAE}

Selaginella denticulata (L.) Spring,

\section{ISOETACEAE}

Isoëtes durieui Bory

\section{ADIANTACEAE}

Adiantum capillus-veneris $\mathrm{L}$.

\section{DENNSTAEDTIACEAE}

Pteridium aquilinum (L.) Kuhn subsp. aquilinum

POLYPODIACEAE

Polypodium cambricum L.

\section{ASPLENIACEAE}

Asplenium onopteris $\mathrm{L}$.

Asplenium trichomanes L. subsp. trichomanes

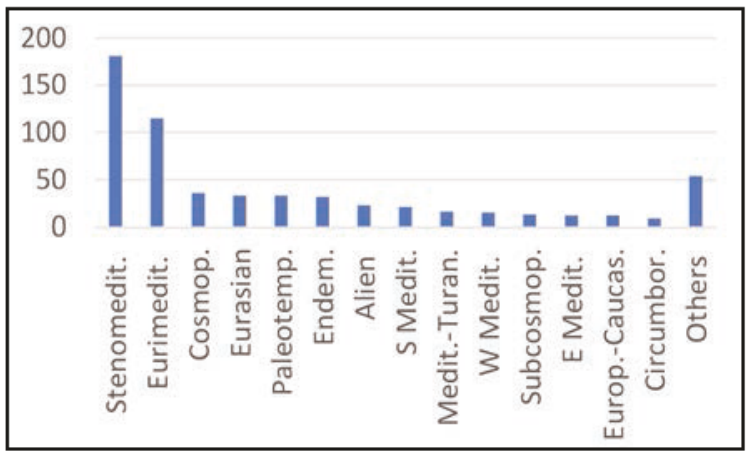

Figure 6. Chorological spectrum of the vascular flora.

Asplenium ceterach L. subsp. ceterach

\section{DRYOPTERIDACEAE}

Dryopteris pallida (Bory) Maire \& Petitm. subsp. pallida

Polystichum setiferum (Forssk.) T. Moore ex Woyn.

\section{SPERMATOPHYTA}

GYMNOSPERMAE

PINACEAE

Pinus pinea L.

\section{ANGIOSPERMAE}

ACANTHACEAE

Acanthus mollis L. subsp. mollis

\section{AMARANTHACEAE}

Achyranthes sicula (L.) All.

*Amaranthus albus L.

*Amaranthus blitum L. subsp. blitum

*Amaranthus cruentus L.

*Amaranthus deflexus L.

*Amaranthus graecizans L. subsp. silvestris (Vill.)

Brenan

*Amaranthus hypocondriacus $\mathrm{L}$.

*Amaranthus retroflexus $\mathrm{L}$.

*Amaranthus viridis $\mathrm{L}$.

\section{AMARYLLIDACEAE}

Allium polyanthum Schult. \& Schult.f.

Allium roseum L. subsp. roseum

Allium sphaerocephalon L. subsp. arvense (Guss.)

Arcang.

Allium subhirsutum L. subsp. subhirsutum

Allium triquetrum $\mathrm{L}$.

*Amaryllis bella-donna $\mathrm{L}$.

Narcissus miniatus Donn.-Morg., Koop. \& Zonn 
Narcissus obsoletus (Haw.) Spach

Narcissus papyraceus Ken Gawl.

Narcissus tazetta L. subsp. tazetta

\section{ANACARDIACEAE}

Pistacia lentiscus $\mathrm{L}$.

Pistacia terebinthus L. subsp. terebinthus

*Rhus coriaria $\mathrm{L}$

\section{APIACEAE}

Ammoides pusilla (Brot.) Breistr.

Anthriscus nemorosa (M.Bieb.) Spreng.

Athamanta sicula L.

Bupleurum fruticosum $\mathrm{L}$.

Bupleurum tenuissimum L.

Chaerophyllum temulum $\mathrm{L}$.

Conium maculatum L.

Daucus carota L. s.l.

Eryngium campestre L.

Eryngium tricuspidatum L. subsp. bocconei (Lam.) Wörz

Eryngium triquetrum Vahl

Foeniculum vulgare Mill subsp. piperitum (Ucria) Bég.

Foeniculum vulgare Mill. subsp. vulgare

Kundmannia sicula (L.) DC.

Oenanthe globulosa $\mathrm{L}$.

Oenanthe pimpinelloides $\mathrm{L}$.

Pimpinella anisoides V. Brig.

Pimpinella gussonii Bertol.

Scandix pecten-veneris L. subsp. pecten-veneris

Smyrnium olusatrum L.

Smyrnium perfoliatum L. subsp. perfoliatum

Thapsia garganica L. subsp. garcanica

Tordylium apulum $\mathrm{L}$.

Torilis arvensis (Huds.) Link subsp. arvensis

Torilis nodosa (L.) Gaertn. subsp. nodosa

\section{APOCYNACEAE}

Vinca major L. subsp. major

\section{ARACEAE}

Arisarum vulgare O.Targ.Tozz. subsp. vulgare

Arum italicum Mill. subsp. italicum

Biarum tenuifolium (L.) Schott subsp. tenuifolium

ARALIACEAE

Hedera helix L. subsp. helix

ASPARAGACEAE

Asparagus acutifolius $\mathrm{L}$.
Bellevalia dubia (Guss.) Kunth subsp. dubia

Charybdis pancration (Steinh.) Speta

Loncomelos narbonensis (L.) Raf.

Muscari comosum (L.) Mill.

Muscari commutatum Guss.

*Oncostema elongatum (Parl.) Speta

Ornithogalum montanum Cirillo ex Ten.

Prospero autumnale (L.) Speta

Ruscus aculeatus L.

\section{ASPHODELACEAE}

Asphodeline lutea (L.) Rchb.

Asphodelus fistulosus $\mathrm{L}$.

Asphodelus ramosus L. subsp. ramosus

\section{ASTERACEAE}

Achillea ligustica All.

Anthemis arvensis L. subsp. arvensis

Anthemis cupaniana Tod. ex Nyman

Arctium minus (Hill) Bernh.

Artemisia arborescens (Vaill.) L.

Bellis anпиa L. subsp. аппиа

Bellis perennis $\mathrm{L}$.

Bellis sylvestris Cirillo

Calendula arvensis (Vaill.) L.

Carduus argyroa Biv.

Carduus pycnocephalus L. subsp. pycnocephalus

Carlina gummifera (L.) Less.

Carlina involucrata Poir.

Carlina lanata $\mathrm{L}$.

Carlina sicula Ten. subsp. sicula

Carthamus lanatus $\mathrm{L}$.

Centaurea calcitrapa $\mathrm{L}$.

Centaurea sicula L.

Centaurea solstitialis L. subsp. schouwii (DC.) Gugler

Cichorium intybus L.

Cirsium creticum (Lam.) d'Urv. subsp. triumfetti (Lacaita) K. Werner

Cirsium scabrum (Poir.) Bonnet \& Barratte

Cirsium vulgare (Savi) Ten. subsp. vulgare

Coleostephus myconis (L.) Cass. ex Rchb.f.

Crepis vesicaria subsp. hyemalis (Biv.) Babc.

Crupina crupinastrum (Moris) Vis.

Cynara cardunculus L. subsp. cardunculus

Dittrichia graveolens (L.) Greuter

Dittrichia viscosa (L.) Greuter subsp. viscosa

Doronicum orientale Hoffm.

Echinops siculus Strobl

Eclipta prostrata (L.) L.

*Erigeron bonariensis $\mathrm{L}$.

Eupatorium cannabinum L. 
Filago eriocephala Guss.

Filago germanica (L.) Huds.

Filago pygmaea $\mathrm{L}$.

Filago pyramidata $\mathrm{L}$.

Galactites tomentosus Moench

*Galinsoga parviflora Cav.

Glebionis coronaria (L.) Spach

Glebionis segetum (L.) Fourr.

Hedypnois rhagadioloides (L.) F.W. Schmidt

Helminthotheca aculeata (Vahl) Lack subsp. aculeata

Helminthotheca echioides (L.) Holub

Hieracium racemosum Waldst. \& Kit. ex Willd. subsp. crinitum $(\mathrm{Sm}$.) Rouy

Hyoseris radiata $\mathrm{L}$.

Hypochaeris achyrophorus $\mathrm{L}$.

Hypochaeris radicata $\mathrm{L}$.

Lactuca sativa L. subsp. serriola (L.) Galasso, Banfi, Bartolucci \& Ardenghi

Lactuca viminea (L.) J. Presl \& C. Presl subsp. viminea

Lapsana communis L. subsp. communis

Leontodon tuberosus $\mathrm{L}$.

Logfia gallica (L.) Cosson \& Germ.

Mycelis muralis (L.) Dumort. subsp. muralis

Notobasis syriaca (L.) Cass.

Onopordum illyricum L.

Pallenis spinosa (L.) Cass. subsp. spinosa

Pentanema squarrosum (L.) D. Gut. Larr., Santos-

Vicente, Anderb., E. Rico \& M.M. Mart.Ort.

Picris hieracioides L.

Pulicaria dysenterica (L.) Bernh.

Pulicaria odora (L.) Rchb.

Reichardia picroides (L.) Roth

Scolymus grandiflorus Desf.

Scolymus maculatus L.

Scorzonera hirsuta (Gouan) L.

Senecio leucanthemifolius Poir. subsp. leucanthemifolius

Senecio lividus L.

Senecio vulgaris L. subsp. vulgaris

Silybum marianum (L.) Gaertn.

Sonchus asper (L.) Hill subsp. asper

Sonchus oleraceus $\mathrm{L}$

*Symphyotrichum squamatum (Spreng.) G.L. Nesom

Taraxacum minimum (V.Brig.) N. Terracc

Taraxacum officinale (L.) Weber ex F.H. Wigg.

Tragopogon porrifolius $\mathrm{L}$.

Urospermum dalechampii (L.) F.W. Schmidt

Urospermum picroides (L.) Scop. ex F.W. Schmidt

Xanthium italicum Moretti

*Xanthium spinosum $\mathrm{L}$.
BORAGINACEAE

Aegonychon purpurocaeruleum (L.) Holub

Anchusa azurea Mill.

Borago officinalis $\mathrm{L}$

Cerinthe major L. subsp. major

Cynoglossum creticum Mill.

Echium italicum L. subsp. siculum (Lacaita) Greuter \& Burdet

Echium plantagineum $\mathrm{L}$.

Heliotropium europaeum $\mathrm{L}$.

Myosotis ramosissima Rochel subsp. ramosissima

Myosotis sylvatica Hoffm. subsp. elongata (Strobl)

Grau

Symphytum gussonei F.W. Schultz

\section{BRASSICACEAE}

Arabidopsis thaliana (L.) Heynh.

Arabis collina Ten. subsp. collina

Arabis collina Ten. subsp. rosea (DC.) Minuto

Arabis hirsuta (L.) Scop.

Arabis verna (L.) R. Br.

Biscutella maritima Ten.

Brassica incana Ten.

Brassica rapa L. subsp. campestris (L.) A.R. Clapham

Capsella bursa-pastoris (L.) Medik. subsp. bursapastoris

Cardamine hirsuta $\mathrm{L}$.

Diplotaxis erucoides (L.) DC. subsp. erucoides

Draba verna L. subsp. praecox (Steven) Rouy \& Foucaud

Draba verna L. subsp. verna

Isatis canescens DC.

Lobularia maritima (L.) Desv.

Lunaria аппиа $\mathrm{L}$.

Raphanus raphanistrum L. subsp. raphanistrum

Sinapis pubescens $\mathrm{L}$. subsp. pubescens

\section{CACTACEAE}

Opuntia ficus-indica (L.) Mill.

CAMPANULACEAE

Campanula dichotoma $\mathrm{L}$.

Campanula erinus $\mathrm{L}$.

Jasione montana L.

CANNABACEAE

Celtis australis L. subsp. australis

CAPPARACEAE

Capparis spinosa $\mathrm{L}$. 


\section{CAPRIFOLIACEAE}

Lonicera implexa Aiton subsp. implexa

\section{CARYOPHYLLACEAE}

Arenaria leptoclados (Rchb.) Guss. subsp. leptoclados Arenaria serpyllifolia L. subsp. serpyllifolia

Cerastium brachypetalum Desp. ex Pers. subsp. brachypetalum

Cerastium glomeratum Thuill.

Cerastium semidecandrum $\mathrm{L}$.

Dianthus siculus C. Presl

Eudianthe coeli-rosa (L.) Endl.

Petrorhagia dubia (Raf.) G. López \& Romo

Petrorhagia illyrica (L.) P.W. Ball \& Heywood subsp. haynaldiana (F.N.Williams) P.W.Ball \& Heywood

Petrorhagia prolifera (L.) P.W. Ball \& Heywood Petrorhagia saxifraga (L.) Link subsp. gasparrinii (Guss.) Greuter \& Burdet

Petrorhagia saxifraga (L.) Link subsp. saxifraga

Polycarpon tetraphyllum (L.) L. subsp. diphyllum

(Cav.) O. Bolós \& Font Quer
Polycarpon tetraphyllum (L.) L. subsp. tetraphyllum Sabulina tenuifolia (L.) Rchb. subsp. tenuifolia

Sagina apetala Ard. subsp. apetala

Silene colorata Poir.

Silene conica $\mathrm{L}$.

Silene fruticosa $\mathrm{L}$.

Silene fuscata Brot.

Silene gallica $\mathrm{L}$.

Silene italica (L.) Pers. subsp. sicula (Ucria) Jeanm.

Silene latifolia Poir.

Silene nocturna $\mathrm{L}$. subsp. nocturna

Silene vulgaris (Moench) Garcke subsp. tenoreana (Colla) Soldano \& F. Conti

Silene vulgaris (Moench) Garcke subsp. vulgaris

Stellaria neglecta Weihe subsp. cupaniana (Jord. \&

Fourr.) Gutermann

Stellaria neglecta Weihe subsp. neglecta

Stellaria pallida (Dumort.) Crép.

\section{CHENOPODIACEAE}

Chenopodium album L. subsp. album

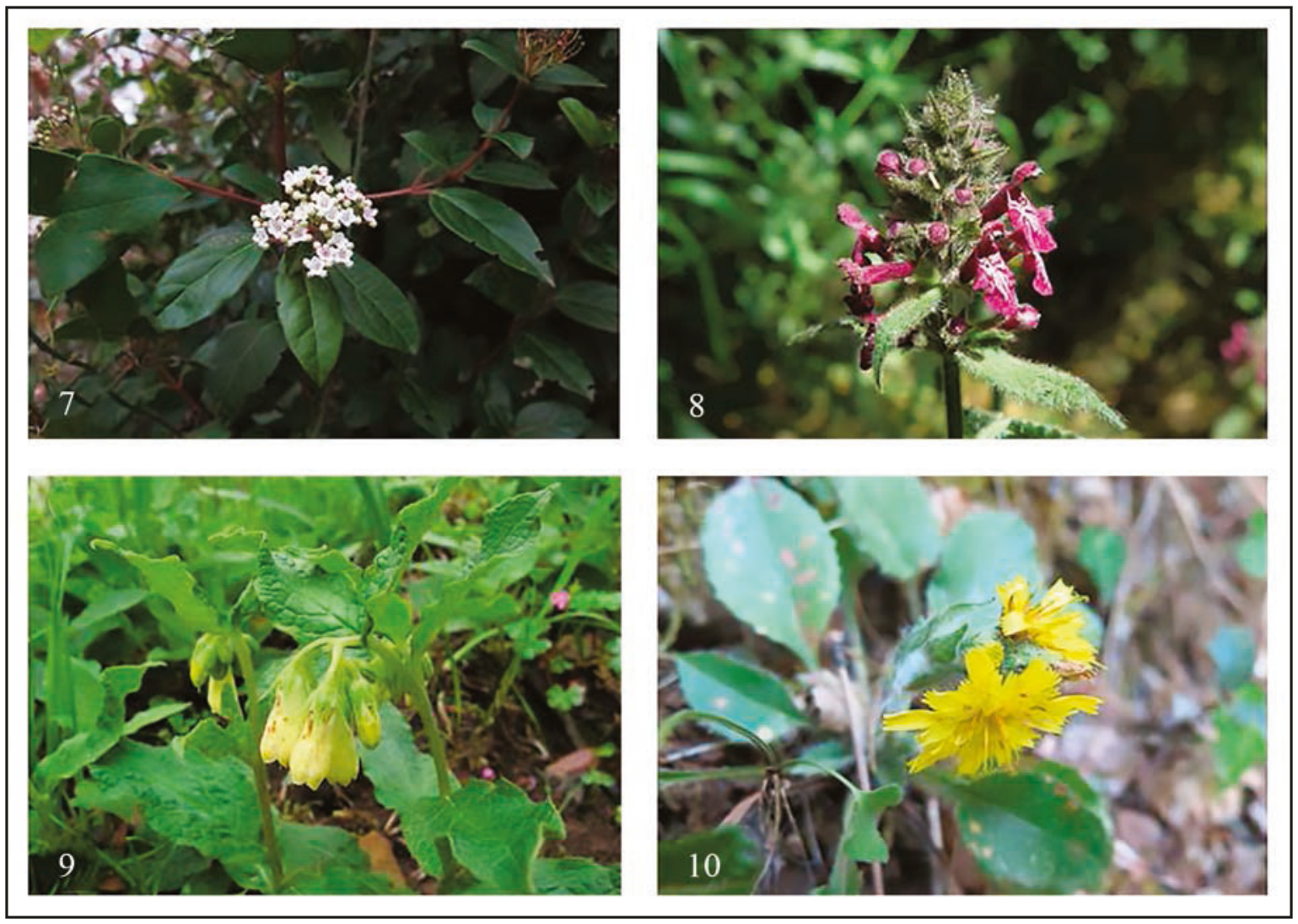

Figure 7. Viburnum tinus subsp. tinus. Figure 8. Stachys sylvatica.

Figure 9. Symphytum gussonei. Figure 10. Hieracium racemosum subsp. crinitum. 
Chenopodiastrum murale (L.) S. Fuentes, Uotila \& Borsch

Chenopodium vulvaria $\mathrm{L}$.

\section{CISTACEAE}

Cistus creticus L. subsp. eriocephalus (Viv.) Greuter \& Burdet

Cistus monspeliensis $\mathrm{L}$.

Cistus salvifolius L.

Tuberaria guttata (L.) Fourr.

\section{COLCHICACEAE}

Colchicum bivonae Guss.

\section{CONVOLVULACEAE}

Convolvulus sepium $\mathrm{L}$.

Convolvulus althaeoides $\mathrm{L}$.

Convolvulus arvensis $\mathrm{L}$.

Convolvulus cantabrica $\mathrm{L}$.

Cuscuta epithymum (L.) L. subsp. epithymum

Ipomoea indica (Burm.) Merr.

\section{CRASSULACEAE}

*Aeonium arboreum (L.) Webb \& Berth.

Petrosedum amplexicaule (DC.) Velayos

Petrosedum sediforme (Jacq.) Grulich

Phedimus stellatus (L.) Raf.

Sedum album L. subsp. album

Sedum caeruleum $\mathrm{L}$.

Sedum cepaea $\mathrm{L}$.

Sedum dasyphyllum $\mathrm{L}$

Umbilicus horizontalis (Guss.) DC.

Umbilicus rupestris (Salisb.) Dandy

\section{CUCURBITACEAE}

Bryonia dioica Jacq.

\section{CYPERACEAE}

Carex caryophyllea Latourr.

Carex distachya Desf.

Carex flacca Schreb. subsp. erythrostachys (Hoppe)

Holub

Carex hallerana Asso

Carex pendula Huds.

Cyperus rotundus $\mathrm{L}$.

Eleocharis palustris (L.) Roem. \& Schult. subsp. palustris

Schoenoplectus lacustris (L.) Palla

Scirpoides holoschoenus (L.) Soják

\section{CYTINACEAE}

Cytinus hypocistis (L.) L.

Cytinus ruber Fourr. ex Fritsch

\section{DIOSCOREACEAE}

Dioscorea communis (L.) Caddick \& Wilkin

\section{DIPSACACEAE}

Dipsacus fullonum L. subsp. fullonum

Sixalix atropurpurea (L.) Greuter \& Burdet

\section{ERICACEAE}

Arbutus unedo $\mathrm{L}$.

Erica arborea L.

\section{EUPHORBIACEAE}

Chrozophora tinctoria (L.) A. Juss.

Euphorbia ceratocarpa Ten.

Euphorbia characias L.

Euphorbia dendroides L.

Euphorbia helioscopia L. subsp. helioscopia

Euphorbia meuselii Geltman

Euphorbia peplus L.

*Euphorbia prostrata Aiton

Euphorbia rigida M. Bieb.

Mercurialis aппиа $\mathrm{L}$.

*Ricinus communis $\mathrm{L}$.

\section{FABACEAE}

Anthyllis vulneraria L. subsp. maura (Beck) Maire Astragalus boeticus L.

Astragalus echinatus Murray

Astragalus hamosus L.

Bituminaria bituminosa (L.) C.H.Stirt.

Cytisus infestus (C. Presl) Guss. subsp. infestus

Cytisus villosus Pourr.

Emerus major Mill. subsp. major

Ervilia hirsuta (L.) Opiz

Hippocrepis multisiliquosa $\mathrm{L}$.

Lathyrus annuus $\mathrm{L}$.

Lathyrus cicera $\mathrm{L}$.

Lathyrus clymenum $\mathrm{L}$.

Lathyrus ochrus (L.) DC.

Lathyrus sphaericus Retz.

Lathyrus sylvestris L. subsp. sylvestris

Lotus biflorus Desr.

Lotus edulis $\mathrm{L}$.

Lotus hispidus DC.

Lotus ornithopodioides L.

Lupinus albus $\mathrm{L}$.

Medicago coronata (L.) Bartal.

Medicago doliata Carmign. 
Medicago intertexta (L.) Mill.

Medicago minima (L.) L.

Medicago murex Willd.

Medicago orbicularis (L.) Bartal.

Medicago polymorpha $\mathrm{L}$.

Medicago rugosa Desr.

Medicago truncatula Gaertn.

Onobrychis caput-galli (L.) Lam.

Ononis diffusa Ten.

Ononis mitissima L.

Ornithopus compressus $\mathrm{L}$.

Pisum sativum L.

Scorpiurus subvillosus L.

Spartium junceum L.

Sulla coronaria (L.) Medik.

Trifolium angustifolium $\mathrm{L}$.

Trifolium arvense L. subsp. arvense

Trifolium bivonae Guss.

Trifolium campestre Schreb.

Trifolium fragiferum L. subsp. fragiferum

Trifolium incarnatum L. subsp. molinerii (Balb. ex Hornem.) Ces.
Trifolium lappaceum $\mathrm{L}$.

Trifolium nigrescens Viv. subsp. nigrescens

Trifolium pallidum Waldst. \& Kit.

Trifolium physodes $\mathrm{M}$. Bieb.

Trifolium pratense $\mathrm{L}$. subsp. pratense

Trifolium repens $\mathrm{L}$.

Trifolium resupinatum $\mathrm{L}$

Trifolium scabrum L.

Trifolium stellatum $\mathrm{L}$.

Trifolium subterraneum L. subsp. subterraneum

Trigonella italica (L.) Coulot \& Rabaute

Trigonella sulcata (Desf.) Coulot \& Rabaute

Tripodion tetraphyllum (L.) Fourr.

Vicia bithynica (L.) L.

Vicia dasycarpa Ten.

Vicia disperma $\mathrm{DC}$.

Vicia hybrida $\mathrm{L}$.

Vicia sativa $\mathrm{L}$.

\section{FAGACEAE}

Castanea sativa Miller

Quercus ilex L. subsp. ilex

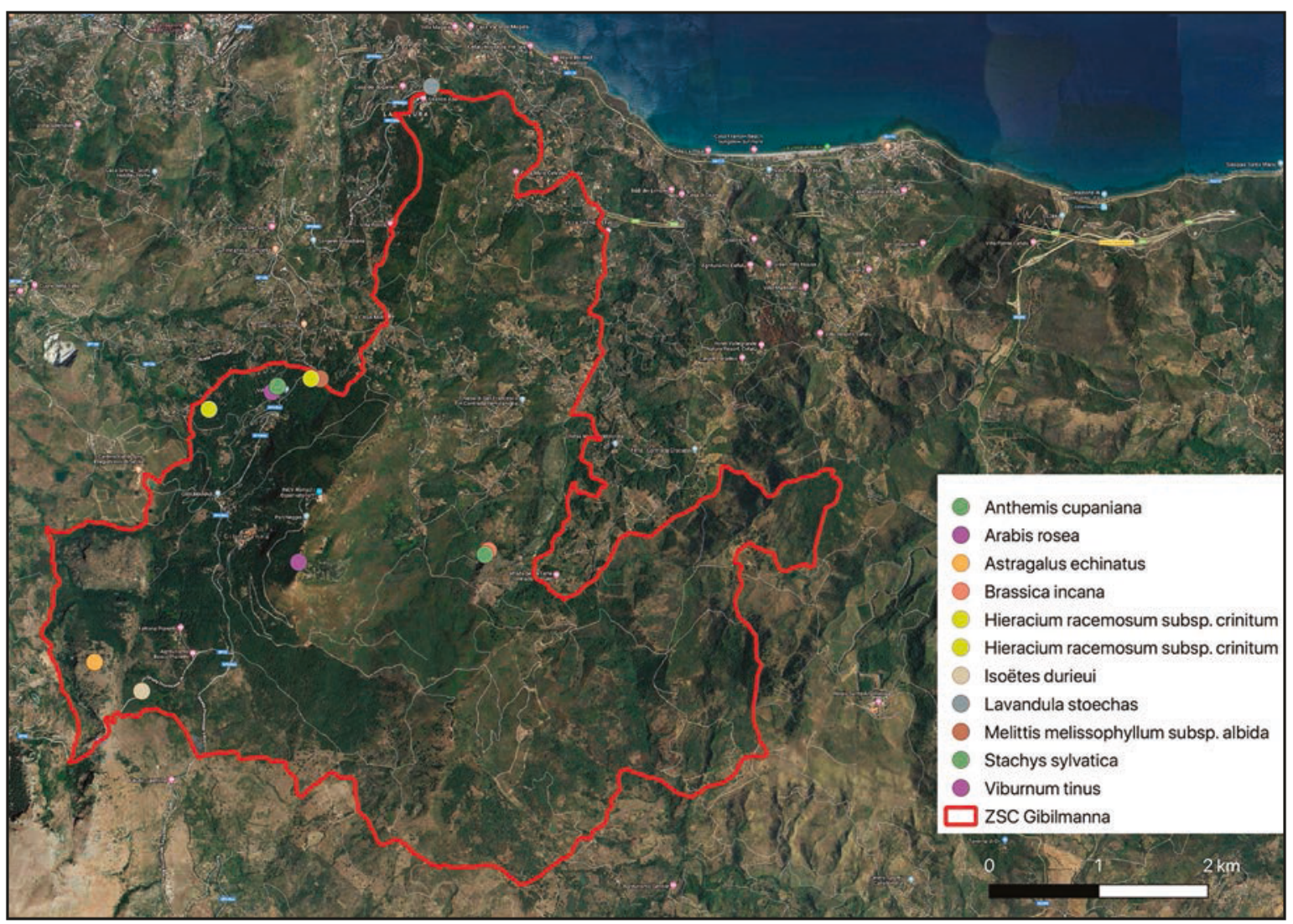

Figure 11. Bosco di Gibilmanna: local distribution of rare taxa or with a phytogeographical interest. 
Quercus suber L.

Quercus virgiliana (Ten.) Ten.

GENTIANACEAE

Blackstonia perfoliata (L.) Huds. subsp. intermedia (Ten.) Zeltner

Centaurium erythraea Rafin subsp. erythraea

Centaurium grandiflorum (Pers.) Ronniger subsp. grandiflorum

Centaurium maritimum (L.) Fritsch

\section{GERANIACEAE}

Erodium ciconium (L.) L'Her.

Erodium cicutarium (L.) L'Her.

Erodium malacoides (L.) L'Her. subsp. malacoides

Geranium dissectum $\mathrm{L}$.

Geranium lucidum L.

Geranium molle L.

Geranium purpureum Vill.

Geranium robertianum $\mathrm{L}$.

Geranium rotundifolium $\mathrm{L}$.

\section{HYPERICACEAE}

Hypericum perfoliatum $\mathrm{L}$.

Hypericum perforatum L. subsp. perforatum

\section{IRIDACEAE}

Crocus longiflorus Raf.

Gladiolus byzantinus Mill.

Gladiolus italicus Mill.

Hermodactylus tuberosus (L.) Mill.

*Iris germanica $\mathrm{L}$.

Juno planifolia (Mill.) Asch.

Moraea sisyrinchium (L.) Ker Gawl.

Romulea bulbocodium (L.) Sebast. \& Mauri

Romulea columnae Sebast. \& Mauri

\section{JUNCACEAE}

Juncus bufonius $\mathrm{L}$.

Juncus effusus L. subsp. effussus

Juncus inflexus L. subsp. inflexus

\section{LAMIACEAE}

Clinopodium nepeta (L.) Kuntze subsp. nepeta

Clinopodium vulgare $\mathrm{L}$. subsp. arundanum (Boiss.)

Nyman

Lamium amplexicaule $\mathrm{L}$.

Lamium bifidum Cirillo subsp. bifidum

Lavandula stoechas L. subsp. stoechas

Marrubium vulgare $\mathrm{L}$.

Melissa officinalis L. subsp. officinalis
Melittis melissophyllum L. subsp. albida (Guss.)

P.W. Ball.

Mentha aquatica L. subsp. aquatica

Mentha pulegium L. subsp. pulegium

Micromeria graeca (L.) Benth. ex Rchb. subsp. graeca

Micromeria nervosa (Desf.) Benth.

Origanum vulgare L. subsp. viridulum (MartinDonos) Nyman

Prunella laciniata (L.) L.

Prunella vulgaris L. subsp. vulgaris

Pseudodictammus hispanicus (L.) Salmaki \& Siadati

Salvia clandestina $\mathrm{L}$.

*Salvia microphylla Kunth

Salvia verbenaca $\mathrm{L}$.

Stachys arvensis (L.) L.

Stachys germanica L. var. dasyanthes (Raf.) Arcang.

Stachys major (L.) Bartolucci \& Peruzzi

Stachys ocymastrum (L.) Briq.

Stachys romana (L.) E.H.L.Krause

Stachys sylvatica L.

Teucrium chamaedrys L. subsp. chamaedrys

Teucrium siculum (Raf.) Guss. subsp. siculum

\section{LAURACEAE}

Laurus nobilis $\mathrm{L}$.

\section{LINACEAE}

Linum strictum L.

Linum tryginum $\mathrm{L}$.

Linum usitatissimum L. subsp. angustifolium (Huds.) Thell.

\section{LYTHRACEAE}

Lythrum junceum Banks \& Sol.

\section{MALVACEAE}

Malva cretica Cav. subsp. cretica

Malva nicaeensis All.

Malva olbia (L.) Alef.

Malva sylvestris $\mathrm{L}$.

Malva trimestris (L.) Salisb.

MORACEAE

Ficus carica $\mathrm{L}$.

NYCTAGINACEAE

*Mirabilis jalapa $\mathrm{L}$. 


\section{OLEACEAE}

Fraximus ornus L. subsp. ornus

Olea europaea L. subsp. oleaster (Hoffmanns. \& Link) Negodi

Phillyrea latifolia $\mathrm{L}$.

ONAGRACEAE

Epilobium hirsutum L.

Epilobium tetragonum L. subsp. tetragonum

\section{ORCHIDACEAE}

Anacamptis collina (Banks \& Sol. ex Russell) R.M. Bateman, Pridgeon \& M.W. Chase

Anacamptis laxiflora (Lam.) R.M. Bateman, Pridgeon \& M.W. Chase

Anacamptis longicornu (Poir.) R.M. Bateman, Pridgeon \& M.W. Chase

Anacamptis papilionacea (L.) R. M. Bateman, Pridgeon \& M.W. Chase subsp. grandiflora (Boiss.) Kreutz 2007

Anacamptis pyramidalis (L.) Rich.

Barlia robertiana (Loisel.) Greuter

Cephalanthera longifolia (L.) Fritsch

Dactylorhiza romana (Sebast.) Soó subsp. markusii

(Tineo) Holub

Dactylorhiza romana (Sebast.) Soó subsp. romana

Epipactis helleborine (L.) Crantz

Epipactis microphylla (Ehrh.) Sw.

Himantoglossum hircinum (L.) Spreng.

Limodorum abortivum (L.) Sw.

Ophrys apifera Huds.

Ophrys archimedea P. Delforge \& M. Walravens

Ophrys bertolonii Moretti subsp. bertolonii

Ophrys bombyliflora Link

Ophrys fusca Link subsp. lupercalis (Devillers-Tersch. \& Devillers) Kreutz

Ophrys lunulata Parl.

Ophrys lutea Cav. subsp. lutea

Ophrys lutea subsp. sicula (Tineo) Soldano 1993

Ophrys pallida Raf.

Ophrys passionis Sennen subsp. garganica E. Nelson

Ophrys speculum Link

Ophrys sphegodes Mill. subsp. panormitana (Tod.)

Kreutz

Ophrys sphegodes Mill. subsp. sphegodes

Ophrys tenthredinifera Willd.

Orchis anthropophora (L.) All.

Orchis brancifortii Biv.

Orchis italica Poir.

Orchis provincialis Balb. ex Lam. \& DC.

Serapias cordigera L. subsp. cordigera
Serapias lingua $\mathrm{L}$.

Serapias nurrica Corrias subsp. nurrica

Serapias vomeracea (Burm. f.) Briq.

Spiranthes spiralis (L.) Chevall.

\section{OROBANCHACEAE}

Bellardia trixago (L.) All.

Bellardia viscosa (L.) Fisch. \& C.A. Mey

Odontites vernus (Bellardi) Dumort. subsp. siculus (Guss.) P.D. Sell

Orobanche caryophyllacea $\mathrm{Sm}$.

Orobanche crenata Forssk.

Orobanche hederae Vaucher ex Duby

Phelipanche lavandulacea (Rchb.) Pomel subsp. lavandulacea

Phelipanche mutelii (F.W. Schultz) Reut.

Phelipanche nana (Reut.) Soják

Phelipanche ramosa (L.) Pomel

OXALIDACEAE

*Oxalis articulata Savigny

*Oxalis pes-caprae $\mathrm{L}$.

\section{PAEONIACEAE}

Paeonia mascula (L.) Miller subsp. russoi (Biv.) Cullen \& Heywood

\section{PAPAVERACEAE}

Fumaria capreolata L. subsp. capreolata

Fumaria flabellata Gasp.

Papaver dubium L.

Papaver hybridum $\mathrm{L}$

Papaver rhoeas L. subsp. rhoeas

Papaver rhoeas L. var. himerense Raimondo \& Spadaro

Papaver setigerum DC.

PLANTAGINACEAE

Antirrhinum majus L. subsp. tortuosum (Bosc ex Lam.) Rouy

Callitriche obtusangula Le Gall

Callitriche stagnalis Scop.

Kickxia spuria (L.) Dumort. subsp. integrifolia

(Brot.) R. Fern.

Linaria purpurea (L.) Mill.

Linaria reflexa (L.) Desf. subsp. reflexa

Misopates orontium (L.) Rafin.

Plantago afra L. subsp. afra

Plantago lagopus $\mathrm{L}$.

Plantago lanceolata $\mathrm{L}$.

Plantago major $\mathrm{L}$. 
Plantago serraria L.

Veronica anagallis-aquatica L. subsp. anagallisaquatica

Veronica cymbalaria Bodard

Veronica persica Poir.

PLUMBAGINACEAE

Plumbago europaea L.

\section{POACEAE}

Aira caryophyllea L.

Ampelodesmos mauritanicus (Poir.) T. Durand \& Schinz

Anisantha madritensis (L.) Nevski subsp. madritensis Anisantha sterilis (L.) Nevski

Anisantha tectorum (L.) Nevski

Anthoxanthum odoratum $\mathrm{L}$.

Arundo donax L.

Arundo plinii Turra

Avena fatua $\mathrm{L}$.

Avena sativa $\mathrm{L}$.

Brachypodium distachyon (L.) P. Beauv.

Brachypodium retusum (Pers.) Beauv. (Host)

Roem. \& Schult.

Brachypodium sylvaticum (Hudson) P.Beauv.

Briza maxima L.

Bromus hordeaceus L. subsp. hordeaceus

Catapodium rigidum (L.) C.E. Hubb. subsp. rigidum Cynodon dactylon (L.) Pers.

Cynosurus cristatus $\mathrm{L}$.

Cynosurus echinatus L.

Dactylis glomerata L. subsp. glomerata

Dactylis glomerata L. subsp. hispanica (Roth) Nyman

Dasypyrum villosum (L.) P. Candargy

Eragrostis cilianensis (All.) Vignolo ex Janch. subsp. cilianensis

Festuca ligustica (All.) Bertol.

Festuca myuros L. subsp. myuros

Hordeum bulbosum L.

Hordeum murinum L. subsp. leporinum (Link) Arcang

Hyparrhenia hirta (L.) Stapf subso. hirta

Koeleria splendens Presl subsp. splendens

Lagurus ovatus L. subsp. ovatus

Lolium perenne $\mathrm{L}$.

Lolium rigidum Gaudin

Melica ciliata L. subsp. ciliata

Oloptum miliaceum (L.) Röser \& H.R. Hamasha

Paspalum distichum $\mathrm{L}$.

Phalaris paradoxa $\mathrm{L}$.
Poa annua L.

Poa infirma Kunth

Poa sylvicola Guss.

Poa trivialis $\mathrm{L}$.

Rostraria cristata (L.) Tzvelev

Sorghum halepense (L.) Pers.

Stipellula capensis (Thunb.) Röser \& H.R. Hamasha

Triticum vagans (Jord. \& Fourr.) Greuter

\section{POLYGONACEAE}

Polygonum aviculare L. subsp. aviculare

Rumex bucephalophorus L. subsp. bucephalophorus

Rumex crispus L.

Rumex cristatus DC.

Rumex thyrsoides Desf

PORTULACACEAE

Portulaca oleracea L.

PRIMULACEAE

Cyclamen hederifolium Aiton subsp. hederifolium

Cyclamen repandum $\mathrm{Sm}$. subsp. repandum

Lysimachia arvensis (L.) U. Manns \& Anderb. subsp. arvensis

Samolus valerandi $\mathrm{L}$.

\section{RANUNCULACEAE}

Anemone hortensis L. subsp. hortensis

Clematis cirrhosa L.

Clematis vitalba $\mathrm{L}$.

Delphinium halteratum Sm. subsp. halteratum

Ficaria verna Huds. subsp. verna

Nigella damascena $\mathrm{L}$.

Ranunculus bulbosus L. subsp. aleae (Willk.) Rouy $\&$ Foucaud

Ranunculus bulbosus L. subsp. neapolitanus (Ten.)

$\mathrm{H}$. Lindb.

Ranunculus bullatus L.

Ranunculus muricatus L.

Thalictrum calabricum Spreng.

RESEDACEAE

Reseda alba $\mathrm{L}$.

RHAMNACEAE

Rhamnus alaternus L. subsp. alaternus 


\section{ROSACEAE}

Agrimonia eupatoria L. subsp. eupatoria

Crataegus monogyna Jacq.

Fragaria vesca L. subsp. vesca

Malus sylvestris (L.) Mill.

Mespilus germanica $\mathrm{L}$.

Potentilla reptans $\mathrm{L}$.

Poterium sanguisorba L. subsp. sanguisorba

Prunus spinosa L. subsp. spinosa

Pyrus communis L. subsp. communis

Pyrus spinosa Forssk.

Rosa canina $\mathrm{L}$.

Rosa micrantha Borrer ex Sm.

Rosa sempervirens $\mathrm{L}$.

Rubus ulmifolius Schott

\section{RUBIACEAE}

Asperula aristata L. f. subsp. scabra Nyman Galium aparine L.

Galium lucidum All.

Galium verrucosum Huds.

Galium verum $\mathrm{L}$. subsp. verum

Rubia peregrina $\mathrm{L}$.

Sherardia arvensis $\mathrm{L}$.

Valantia muralis L.

\section{RUTACEAE}

Ruta chalepensis $\mathrm{L}$.

\section{SALICACEAE}

Salix pedicellata Desf.

SANTALACEAE

Osyris alba L.

\section{SAPINDACEAE}

Acer campestre L.,

\section{SAXIFRAGACEAE}

Saxifraga bulbifera $\mathrm{L}$.

Saxifraga tridactylites $\mathrm{L}$.

\section{SCROPHULARIACEAE}

Scrophularia peregrina $\mathrm{L}$.

Verbascum sinuatum $\mathrm{L}$.

Verbascum thapsus L. subsp. thapsus

\section{SIMAROUBACEAE}

*Ailanthus altissima (Mill.) Swingle
SMILACACEAE

Smilax aspera L.

SOLANACEAE

Mandragora autumnalis Bertol.

Solanum nigrum L.

THYMELAEACEAE

Daphne gnidium L.

Daphne laureola $\mathrm{L}$.

\section{TYPHACEAE}

Typha angustifolia $\mathrm{L}$.

Typha latifolia $\mathrm{L}$.

\section{ULMACEAE}

Ulmus minor Mill. subsp. canescens (Melville) Browicz \& Ziel.

\section{URTICACEAE}

Parietaria judaica L.

Parietaria lusitanica L. subsp. lusitanica

Urtica dioica L. subsp. dioica

Urtica membranacea Poir.

Urtica pilulifera $\mathrm{L}$.

\section{VALERIANACEAE}

Centranthus ruber (L.) DC. subsp. ruber

Fedia graciliflora Fisch. \& C.A. Mey.

Valerianella eriocarpa Desv.

\section{VERBENACEAE}

Verbena officinalis $\mathrm{L}$.

VIBURNACEAE

Viburnum tinus $\mathrm{L}$. subsp. tinus

\section{VIOLACEAE}

Viola alba Besser subsp. dehnhardtii (Ten.) W. Becker

Viola odorata $\mathrm{L}$.

\section{ZYGOPHYLLACEAE}

Tribulus terrestris L.

\section{CONCLUSIONS}

This study allowed to survey the vascular flora of the Gibilmanna and Cefalù woods, an area clas- 
sified as a Special Area of Conservation (S.A.C.). It provides new data on a territory that had not previously been the subject of specific floristic investigations, having a certain phytogeographical interest for its location and ecological features.

Besides, the results obtained show a low incidence of exotic plants (23 species), mostly restricted to cultivated areas and to the most disturbed sites located near country houses and roads.

\section{ACKNOWLEDGEMENTS}

We want to thank for useful suggestions and for providing some unpublished data Prof. Gianniantonio Domina (University of Palermo, Italy) and Dr. Antoine Giardina (Museo naturalistico "F. Minà Palumbo", Castelbuono, Italy).

\section{REFERENCES}

Bartolucci F., Galasso G., Albano A., Alessandrini A., Ardenghi N.M.G., Astuti G., Bacchetta G., Ballelli S., Banfi E., Barberis G., Bernardo L., Bouvet D., Bovio M., Cecchi L., Di Pietro R., Domina G., Fascetti S., Fenu G., Festi F., Foggi B., Gallo L., Gottschlich G., Gubellini L., Iamonico D., Iberite M., JiménezMejías P., Lattanzi E., Marchetti D. Martinetto E., Masin R.R., Medagli P., Passalacqua N.G., Peccenini S., Pennesi R., Pierini B., Poldini L., Prosser F., Raimondo F.M., Roma-Marzio F., Rosati L., Santangelo A., Scoppola A., Scortegagna S., Selvaggi A., Selvi F., Soldano A., Stinca A., Wagensommer R.P., Wilhalm T. \& Conti F., 2018. An updated checklist of the vascular flora native to Italy. Plant Biosystems, 152: 179-303. https://doi.org/10.1080/11263 504.2017. 1419996

Brullo S., 1984. Contributo alla conoscenza della vegetazione delle Madonie (Sicilia settentrionale). Bollettino dell'Accademia Gioenia di Scienze Naturali, Catania, 16: 351-420.

Domina G. \& Mazzola P., 2005. Su un frammento d'erbario di Enrico Pirajno di Mandralisca. Il Naturalista siciliano, 29: 3-17.

Falci A. \& Giardina A., 2004. Parco delle Madonie. Le Orchidee. Paruzzo Editore, Caltanissetta, 96 pp.

Galasso G., Conti F., Peruzzi L., Ardenghi N.M.G., Banfi E., Celesti-Grapow L., Albano A., Alessandrini A,. Bacchetta G., Ballelli S., Bandini Mazzanti M., Bar- beris G., Bernardo L., Blasi C., Bouvet D., Bovio M., Cecchi L., Del Guacchio E., Domina G., Fascetti S., Gallo L., Gubellini L., Guiggi A., Iamonico D., Iberite M., Jiménez-Mejías P., Lattanzi E., Marchetti D., Martinetto E., Masin R.R., Medagli P., Passalacqua N.G., Peccenini S., Pennesi R., Pierini B., Podda L., Poldini L., Prosser F., Raimondo F.M., RomaMarzio F., Rosati L., Santangelo A., Scoppola A., Scortegagna S., Selvaggi A., Selvi F., Soldano A., Stinca A., Wagensommer R.P., Wilhalm T. \& Bartolucci F., 2018. An updated checklist of the vascular flora alien to Italy. Plant Biosystems, 152: 556-592. https://doi.org/10.1080/11263504.2018. 1441197

Greuter W., Burdet H.M. \& Long G., 1984. Med-Checklist.1. Pteridophyta, Gymnospermae, Dicotyledones (Acanthaceae-Cneoraceae). Conservatoire et Jardin botaniques de la Ville de Genève.

Greuter W., Burdet H.M. \& Long G., 1986. Med-Checklist. 3. Dicotyledones (Convolvulaceae-Labiatae). Conservatoire et Jardin botaniques de la Ville de Genève.

Palmeri G., 2007. Gibilmanna, al crepuscolo del mondo contadino. Publisicula, Palermo, 91 pp.

Pignatti S., 1982. Flora d'Italia. 1-3. Bologna, Edagricole.

Pignatti S., 2017-2019. Flora d'Italia, 1-4 vol. Edagricole-New Business Media, Milano.

Raimondo F.M., 1980. Carta della vegetazione di Piano della Battaglia e del territorio circostante (Madonie, Sicilia). Quaderni C.N.R. AQ/1/336: 1-43.

Raimondo F.M., 1984a. La vegetazione rupestre delle Serre di Quacella (Madonie, Sicilia). Atti Società Toscana di Scienze Naturali, 90: 31-41.

Raimondo F.M., 1984b. On the natural history of the Madonie Mountains. Webbia, 38: 29-52. https://doi. org/10.1080/00837792.1984.10670294

Raimondo F.M., Gianguzzi L. \& Ilardi V., 1994. Inventario delle specie "a rischio" nella flora vascolare nativa della Sicilia. Quaderni di Botanica Ambientale Applicata, 3: 65-132.

Raimondo F.M. \& Mazzola P., 1983. Aggiunte alla flora delle Madonie (Sicilia). Atti Accademia Scienze di Palermo, 40: 1-11.

Raimondo F.M., Mazzola P. \& Domina G., 2004b. Check-list of the vascular plants collected during Iter Mediterraneum III. Bocconea, 17: 65- 231.

Raimondo F.M., Schicchi R. \& Surano N., 2004a. Carta del paesaggio e della biodiversità vegetale del parco delle Madonie. Il Naturalista siciliano, 27: 71-137.

Tutin T.G., Heywood V.H., Burges N.A., Moore D.M.,Valentine D.H., Walters S.M. \& Webb D.A., editors. 1964-1980. Flora Europaea (1-5). Cambridge. 